$\begin{array}{ll} & \text { Etnográfica } \\ \text { etnográfica } & \text { Revista do Centro em Rede de Investigação em }\end{array}$

Antropologia

vol. 17 (3) | 2013

Vol. $17(3)$

\title{
Un art sonore situé : le sens et l'espace
}

A situated sound art: meaning and space

\section{Corsin Vogel}

\section{(2) OpenEdition}

Journals

Édition électronique

URL : https://journals.openedition.org/etnografica/3260

DOI : 10.4000/etnografica.3260

ISSN : 2182-2891

\section{Éditeur}

Centro em Rede de Investigação em Antropologia

\section{Édition imprimée}

Date de publication : 30 octobre 2013

Pagination : 605-616

ISSN : 0873-6561

Référence électronique

Corsin Vogel, « Un art sonore situé: le sens et l'espace», Etnográfica [En ligne], vol. 17 (3) | 2013, mis en ligne le 29 octobre 2013, consulté le 09 février 2022. URL : http://journals.openedition.org/etnografica/ 3260 ; DOI : https://doi.org/10.4000/etnografica.3260

\section{(c) (i) (9)}

Etnográfica is licensed under a Creative Commons Attribution-NonCommercial 4.0 International License. 


\section{Un art sonore situé : le sens et l'espace}

\section{Corsin Vogel}

Les installations sonores situées telles que je les conçois utilisent non seulement l'acoustique du lieu, mais également le contenu sonore souvent très diversifié de l'espace public, pour projeter l'auditeur dans de nouveaux espaces perceptifs. Une composition sonore à partir de prises de son in situ et un système de diffusion adapté au lieu me permettent ainsi de travailler sur une démultiplication des espaces, en réactivant les représentations mentales des auditeurs. À partir de certaines de mes créations sonores récentes, je montrerai de quelle façon j'utilise la signification des sons, la matérialisation d'un corps sonore et la spatialisation du son pour construire chacune de ces œuvres.

MOTS-CLÉS : art sonore, installation sonore, perception, sémantique, spatialisation.

A situated sound art: meaning and space - The sound installations I create take benefit from the acoustics of the place but also from the very rich sound content of the public space, in order to bring the listener into new perceptive spaces. A sound composition based on situated sound recordings and with an appropriate sound diffusion system enables us to work on the condensation of spatial complexity, by reactivating mental representations of the listeners. Illustrating my working progress with recent sound creations, I will show the way I use the meaning of sound, the materialization of a sound body and the spatialization of sound in order to build up these sonic works.

KEYWORDS: sound art, sound installation, perception, semantics, spatialization.

VOGEL, Corsin (info@corsinvogel.com) - artiste/compositeur, Dole, France. 


\section{LA RECONNAISSANCE ET LA LOCALISATION DES SONS CONSTITUENT} deux fonctions premières de la perception. En effet, identifier une source sonore, c'est-à-dire lui donner du sens, et la positionner dans l'espace, sont deux fonctions essentielles à la survie. Dans les temps anciens, il s'agissait de se protéger des prédateurs ; aujourd'hui, ce sont par exemple les véhicules motorisés qui représentent un danger pour le piéton ou le cycliste. D’où le besoin de signaux d'urgence univoques et facilement localisables. M'appuyant sur mes recherches en acoustique et en perception auditive, notamment dans le cadre d'un doctorat sur la perception des signaux d'avertissement en contexte urbain, j’ai développé une approche artistique de ces deux fonctions essentielles de la perception auditive consistant à jouer avec la sémantique des sons et avec l'illusion d'une omniprésence, d'une ubiquité des figures associées aux sons. Ces recherches et expérimentations autour de la perception du son comme un signal d'une part, et autour du son comme un objet sémantique de notre environnement quotidien d'autre part, m’ont amené à développer une démarche artistique intimement liée au lieu ou au contexte de production du son.

Ainsi, mes installations in situ explorent et expérimentent les formes sonores et acoustiques singulières d'un lieu, d'un contexte, d'une temporalité particulière. Ces formes sonores, souvent considérées comme gênantes car quotidiennes et anecdotiques, sont pourtant d'une grande richesse, tant du point de vue des sources que des ambiances, et elles peuvent être mises en valeur en les sortant de leur contexte d'origine afin de redonner de la musicalité aux sons du quotidien. Cette approche s'inscrit donc dans le prolongement de la musique concrète et notamment du travail du compositeur Luc Ferrari (Caux 2009). J'enregistre des sons que je retravaille, détourne, déforme, recompose pour créer des objets sonores inédits qui révèlent la richesse, la complexité, le pouvoir d'évocation et d'interprétation des formes et des textures sonores.

Dans la mise en espace de mes installations sonores, le lieu et son acoustique s'avèrent primordiaux, la technique de diffusion devant permettre au visiteur de se projeter dans de nouveaux espaces imaginaires, en mettant en évidence les propriétés acoustiques du lieu (comme dans certaines œuvres de Janet Cardiff et George Bures Miller) ou, au contraire, en créant des espaces sonores radicalement différents de ceux attendus dans le lieu (comme par exemple dans certaines installations de Bill Fontana).

Par ailleurs, lorsqu'elles ne sont pas associées à un lieu, mes compositions sonores sont situées dans une forme sonore préexistante : enregistrement audio récent ou archive sonore, œuvre littéraire, objet sonore abstrait ou naturaliste... Cette forme constitue alors une contrainte de départ et un matériau souvent exclusif pour élaborer une réinterprétation de la forme sonore. La réinterprétation d'enregistrements sonores préexistants devient ainsi la base de mon schéma narratif et de mon écriture musicale électroacoustique ou mixte. Elle autorise une grande liberté de transformation, de malaxage de la forme 
sonore et constitue une démarche artistique inscrite plutôt dans une temporalité que dans une spatialité.

J'aborderai ici trois aspects qui me paraissent essentiels à la création sonore in situ: la sémantique des sons et leurs représentations mentales associées, la spatialisation du son dans sa dimension à la fois immersive et d'espace de projection, et enfin, la matérialité du corps sonore comme objet palpable ayant une épaisseur, disposé dans un espace d'exposition interagissant avec lui.

\section{LA SÉMANTIQUE DES SONS}

Depuis 1995, je travaille sur la perception du son et sur la puissance sémantique et plastique de la matière sonore. La sémantique tout d'abord: une thèse de doctorat sur la perception auditive, plus précisément sur l'identification des signaux d'avertissement en contexte urbain (Vogel 1999), m'a permis d'envisager le son en donnant une place centrale à l'auditeur et non à la source sonore, comme cela se fait en physique. ${ }^{1}$ Les travaux sur la catégorisation prototypique (Rosch 1978) et sur la signification des sons (Dubois 1993, 1998 [1993]) m'ont permis d'appréhender le lien entre les représentations dimensionnelles de la physique et la perception catégorielle étudiée en psychologie cognitive. En effet, le concept de catégorisation prototypique suppose que les traitements perceptifs relatifs à des stimuli aboutissent nécessairement à l'élaboration de catégories. Deux principes fondamentaux régissent la formation des catégories: d'une part, l'économie cognitive soutient le fait que l'être humain cherche à obtenir un maximum d'informations de son environnement avec un minimum d'efforts cognitifs; d'autre part, la structure du monde perçu suppose l'existence de corrélats entre les propriétés des objets qui composent le monde physique, ce qui conduit à une réduction notable du nombre de caractéristiques nécessaires à l'individu pour comprendre le monde : on parle de propriétés pertinentes. A l'intérieur de chaque catégorie existe une forme moyenne, le prototype, qui réunit les propriétés communes aux objets. Cette forme prototypique se définit par l'intermédiaire de ses rapports de ressemblance et de dissemblance avec les autres objets du monde perçu et permet d'introduire la notion de typicalité d'un objet, de par sa relation au prototype (Dubois 1993: 280-281). Le sens lié à un son donné sous-entend une identification de la source sonore, mais celle-ci peut se faire de différentes façons en fonction du contexte d'écoute. Il y a alors ambiguïté d'identification selon le contexte.

Mon travail sur la sémantique des sons consiste notamment à opérer des transformations sonores pour accéder à diverses représentations mentales auprès des auditeurs. La matière et les objets sonores tels qu'ils ont été définis

l Thèse présenté au Laboratoire d'Acoustique Musicale, Paris (Université Pierre et Marie Curie, CNRS, Ministère de la Culture), aujourd'hui rebaptisé laboratoire Lutheries - Acoustique - Musique. 
et composés par les fondateurs de la musique concrète, Pierre Schaeffer et Luc Ferrari notamment, en constituent la base. Ainsi, je me concentre en premier lieu sur le timbre sonore, la reconnaissance - ou non - des sources, leurs couleurs sonores et leurs évolutions narratives. Cette approche s'articule autour de la question du sens véhiculé, suggéré, par les sons, et en particulier, la recherche de cette limite perceptive entre l'objet sonore naturel (par exemple la voix parlée) et l'objet musical inouï (la même voix déformée au point d'être perçue comme un nouveau son instrumental original). Certaines de mes compositions électroacoustiques sont réalisées à partir d'un unique élément sonore enregistré dans un contexte spécifique donnant sens à l'œuvre: un "clic" analogique et un "clic" numérique dans "Rencontre du troisième Clic" (2007), ou encore les dix "mots de tonnerre" de Finnegans Wake de James Joyce (1939) récités par Thomas Douglas dans "Wylerhafen” (2000). D’autres œuvres mélangent des sonorités et une écriture instrumentale plus formelle avec des objets musicaux inouiis : la voix et la guitare dans "Borgonovo via Paris" (2007), ou bien le violoncelle dans "Cheminements" (2008).

Pour concevoir l'installation "Lire Freud... Ecouter Freud - La voix de Sigmund Freud réinterprétée" exposée à la Bibliothèque Sigmund Freud à Paris (2011), je me suis emparé d'un matériau mythique: la voix de Sigmund Freud, dans un enregistrement réalisé par la BBC à Londres en 1938. Seul enregistrement connu de la voix de Freud, ce matériau brut possède à lui seul un pouvoir de fascination et d'interprétation que j'ai souhaité utiliser pour en proposer de nouvelles représentations soumises à la libre appropriation et interprétation de chaque visiteur.

Une première image, visuelle, de la voix de Sigmund Freud est présentée dans la vitrine de la bibliothèque (figure 1 ). Cette transcription sonagraphique ${ }^{2}$ d'un extrait de l'enregistrement ("I succeeded in acquiring pupils and building up an international psycho-analytic association") évoque la voix et la personne de Freud avec une matérialité, une physicalité d'autant plus troublante qu'elle demeure en même temps abstraite, résistant à la lecture immédiate pour susciter l'interprétation ou la contemplation.

Une autre image, sonore celle-là, de la voix de Sigmund Freud, est ensuite à découvrir dans l'entrée de la bibliothèque. En respectant scrupuleusement la temporalité de la prosodie de Freud et en utilisant comme unique matériau sonore l'intégralité de l'enregistrement de la BBC, je propose, à partir du discours de Freud, une réflexion sur la notion d'interprétation. La voix de Freud, très distincte et présente au début, avec la sonorité de l'enregistrement d'époque, glisse petit à petit vers une musicalité plus abstraite, tout d'abord par une démultiplication et une aliénation de la voix et du langage. Puis, des 
dans la ville, mais plutôt de reconstituer une forme sonore qui évoque un passé acoustique et réactive les représentations en mémoire des visiteurs. En effet, les sources sonores présentées ont disparu du paysage local depuis plusieurs décennies.

\section{LA SPATIALISATION DU SON/ / L'IMMERSION SONORE}

L'espace lié au son constitue un deuxième axe de réflexion de mon travail de création. Au cours de mon doctorat, une autre problématique expérimentale est apparue: celle de la diffusion d'échantillons sonores

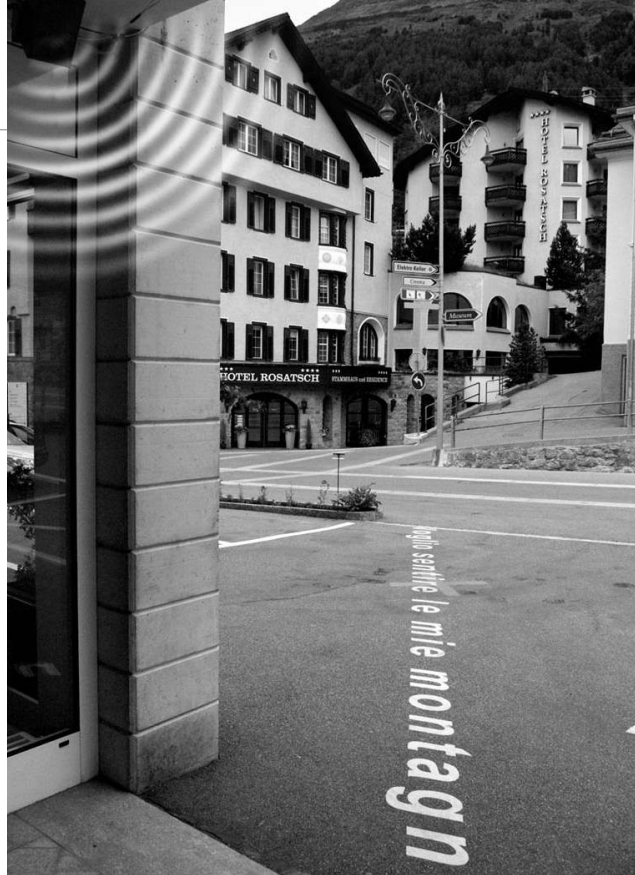

Figure 2 - "Voglio sentire le mie montagne". Vias d'Art Puntraschigna 2011, Pontresina, Suisse. Photo : Corsin Vogel. urbains sur des haut-parleurs et dans un laboratoire. Lapproche choisie ne fut pas celle de la réalité virtuelle où l'on essaie de s'approcher au maximum de la réalité physique du son et de sa propagation, mais celle de la validité écologique des sons (Gibson 1986 [1979]) pour laquelle on doit s'assurer que les auditeurs réagissent comme si ils étaient en situation réelle. Gibson considère que la perception doit s'étudier dans des conditions naturelles, écologiques, en accord avec nos mouvements dans notre environnement. Par ailleurs, il introduit le concept d'affordance, qui correspond à une information potentiellement utile et pertinente pour agir. Ainsi, l'individu utilise un certain nombre de ces capacités pour les mettre en relation avec son environnement. L'accent est donc porté sur les descriptions de la structure des événements pertinents pour les auditeurs, d'où émergent certaines propriétés perçues comme invariantes. Le concept de validité écologique nous apporte les moyens méthodologiques pour effectuer certaines abstractions du monde sensible sans pour autant altérer l'accès aux représentations en mémoire des individus. Il s'agit donc de faire fonctionner l'illusion référentielle des auditeurs à l'aide des sons enregistrés, abstraits d'un environnement multisensoriel, en donnant les informations nécessaires pour atteindre les mêmes représentations en mémoire que les auditeurs avaient établies lors d'une perception directe, en situation. S'assurer de l'adéquation entre la réaction des sujets en laboratoire et celle au quotidien consiste alors à tester l'ensemble de la chaîne sonore, de l'enregistrement à la restitution. Le système le plus efficace sera celui qui permet aux individus de s'intégrer dans la séquence sonore avec l'équipement technique le plus léger possible. Il fera l'objet de tests d'écoute pour valider expérimentalement les techniques 
de prise de son, ainsi que les configurations de diffusion sonore dans un espace d'écoute donné.

Pour des ambiances sonores urbaines, immersives par essence, la spatialisation du son devient une thématique incontournable. Mais la notion d'installation sonore, située dans une partie d'un espace public, nécessite d'aborder la question de l'intérieur et de l'extérieur de l'installation. Si l'intérieur fait référence à l'immersion auditive, l'extérieur met en avant le concept de corps sonore, d'objet sonore appréhendé comme un volume palpable qui évolue dans le temps. J'y reviendrai dans la dernière partie.

Je cherche, dans la mesure du possible, à restituer cette immersion sonore (ou enveloppement) dans mes créations. Plusieurs possibilités s'offrent à nous : travailler sur la base d'une diffusion multicanal (sur quatre ou six haut-parleurs, voire plus), profiter d'un espace réverbérant ou aux propriétés acoustiques particulières, ou encore jouer avec la capacité d'abstraction des auditeurs: plus un son a une sémantique associée forte, plus on se projette facilement dans ce son. L'absence visuelle des sources sonores confère à la spatialisation du son un effet de surprise, une attention particulière, là où le cinéma se heurte à des problèmes de cohérence avec l'image (Bailblé 1998). Par ailleurs, l'architecture du lieu et son implantation dans l'espace environnant prédéterminent le type de diffusion et la forme du corps sonore souhaités, tout en constituant des contraintes acoustiques qui permettent de réinterpréter le lieu et de lui donner des significations différentes.

Les expérimentations sur la validité écologique des sons m’ont amené à développer des systèmes de diffusion du son en multicanal, spécifiquement adapté au lieu. Ainsi, l'œuvre monumentale “Rouge Ruisseau” (2008), pour laquelle il s'agissait de sonoriser une rue sur une centaine de mètres, a nécessité 16 haut-parleurs répartis régulièrement de part et d'autre de la rue, comme huit systèmes stéréophoniques dépendants les uns des autres (figure 3). J'ai retenu ce système pour créer l'illusion d'une rivière sonore dévalant la rue et allant s'engouffrer dans une librairie dans laquelle se tenaient au même moment des lectures de romans noirs. Les sons, issus en particulier de chants révolutionnaires de la Commune, défilent, telle une manifestation du souvenir dans cette rue où furent érigées les dernières barricades de la Commune et qui, bien plus tôt, accueillait un canal d'approvisionnement de Paris en eau potable. Lapproche est donc historique, sans enregistrements sonores et j'ai essayé d'inciter les passants à se laisser entraîner par ce flot sonore délimité par l'architecture d'une rue très enveloppante et chargée d'histoire, pour servir les lectures d'extraits de romans noirs.

L'installation "Hors-Champs" (2011) a été pensée pour une bergerie dans laquelle le son était diffusé: quatre enclos délimitant les espaces réservés à quatre troupeaux de moutons m'ont conduit à utiliser une quadriphonie installée dans la charpente, les haut-parleurs étant orientés vers l'extérieur, vers 


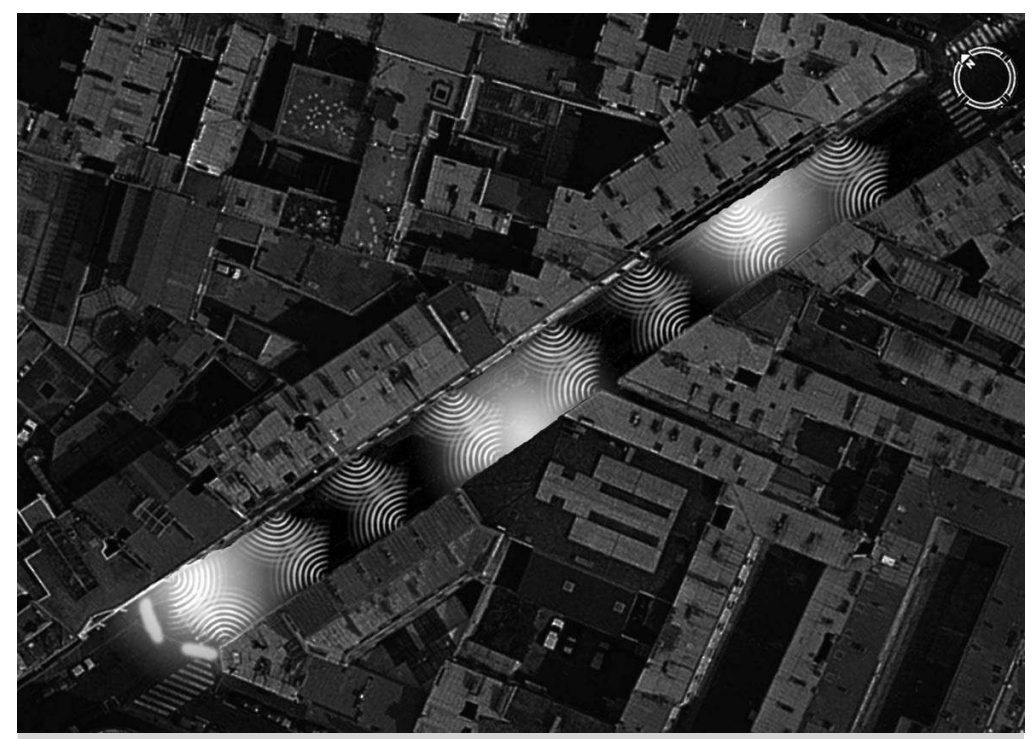

Figure 3 - "Rouge Ruisseau”. Nuit Blanche, 2008, Paris, France. Photo: Corsin Vogel.

les quatre enclos (figure 4). L'écoute se faisait donc en général indirectement, le son se réfléchissant sur les murs et le sol des quatre coins de la ferme, sauf à se positionner dans l'un des quatre enclos à brebis. Un visiteur se trouvant au centre de la bergerie profitait ainsi d'une quadriphonie avec des sources se déplaçant tout autour de lui, tandis qu'un auditeur excentré pouvait identifier quatre corps sonores associés aux quatre parties distinctes de la bergerie. Il s'agissait de proposer une écoute décalée de l'espace intérieur de la bergerie de Vernand: un hors-champ spatial, cinématographique, dans lequel on perçoit la présence des brebis pourtant visuellement absentes, et un hors-champ temporel, qui rappelle l'ambiance sonore de la bergerie durant les mois d'hiver.

\section{LA MATÉRIALITÉ DU CORPS SONORE}

Dans son Traité des objets musicaux, Schaeffer (1966) définit l'objet sonore comme un "élément sonore fini ayant un début et une fin, et de forme émergeant sur un fond". Cette définition peut être également associée à la notion plus générale d'événement sonore. Theile (1980) tente d'être un peu plus précis en ajoutant à la structure de l'événement sonore, définie ci-dessus, un lieu et une source: c'est "la partie du son provenant d'une source sonore unique et qui détermine ou influence les propriétés de l'événement auditif associé, à savoir un lieu et une structure". Mais l'objet sonore a également une matérialité, une masse, une épaisseur, un grain, une profondeur, un peu 


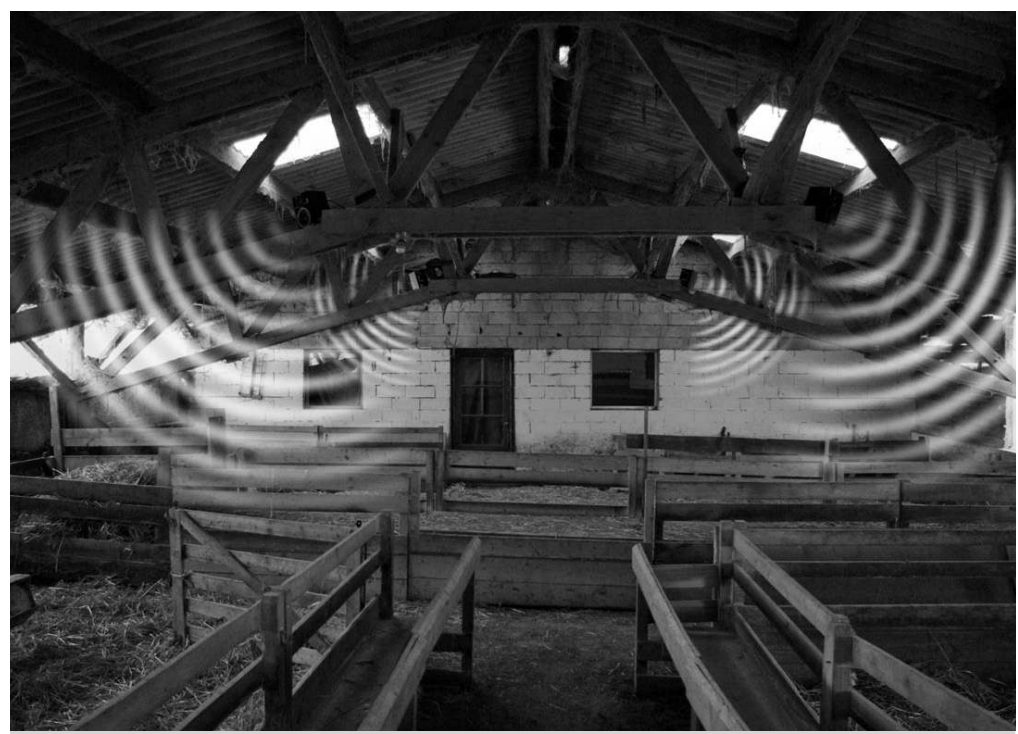

Figure 4 - "Hors-Champs". Troisième cycle d'art contemporain, 2011 , Vernand, France. Photo: Corsin Vogel.

comme une photographie (Deshays 2004). Deshays précise que le son est l'interprétant de l'image filmique, mais que, placé aux côtés d'un objet immobile ou dans un espace architectural donné, il en modifie la perception.

C'est le cas de l'installation "Schamser Klangskulptur" (2010, Zillis, Suisse) dont le système de diffusion est basé sur un hexagone régulier aux sommets duquel se trouvent six haut-parleurs (figure 5). Ce système hexaphonique est le premier d'une série de pièces travaillées dans ce format. Il s'agit pour moi de disposer d'un système minimal permettant de restituer avec précision suffisante un espace sonore (en termes de localisation auditive écologique, qui autorise une illusion auditive cohérente pour le visiteur, et non de réalité virtuelle, qui cherche à être proche de la propagation physique du son). Au centre du cercle formé par ces six haut-parleurs, l'immersion sonore permet à l'auditeur de faire abstraction des murs, de se projeter à l'extérieur du bâtiment et de "goûter" aux sonorités de toute la vallée de Schams. A l'extérieur du cercle, c'est un corps sonore qui se présente devant nous, palpable et invitant le spectateur à le pénétrer et à le traverser. L'installation propose une approche "anecdotique" (selon le compositeur Luc Ferrari) de la vie dans la vallée de Schams. Des prises de son in situ alimentent une composition spatialisée dans laquelle l'auditeur peut s'immerger pour se projeter dans de nouveaux espaces imaginaires. Le lieu de diffusion est un espace confiné, petit, voûté et par conséquent à l'acoustique très particulière. L'idée était donc de placer le visiteur au centre du système hexaphonique et de lui permettre "d'abattre" 


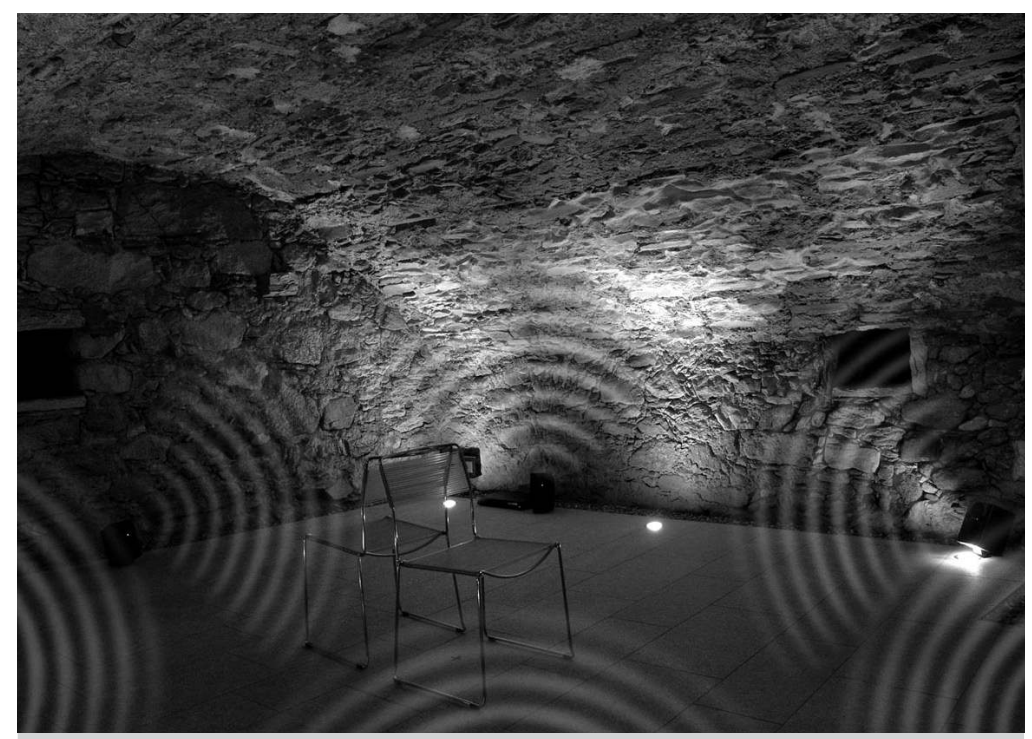

Figure 5 - “Schamser Klangskulptur”. 555 Ons Val Schons, 2010, Zillis, Suisse. Photo: Corsin Vogel.

les murs et de se projeter à l'extérieur par simple immersion dans l'espace sonore créé. Il peut ainsi promener son imaginaire dans toute la vallée et sur les alpages qui l'entourent, malgré l'obstruction visuelle des murs de la salle, et écouter le fauchage et le séchage de l'herbe, la traite des vaches ou encore une déambulation dans la maison de retraite voisine. L’ouvre diffusée se mélange continuellement aux sources sonores qui passent (voitures, enfants...), que l'on peut entendre par une petite fenêtre et qui modifient à chaque écoute notre perception de l'installation. Une incitation à réfléchir à l'environnement sonore et aux activités de la vallée, en les écoutant autrement.

Une autre installation sonore, "Wandelgang" (2011), reprend cette idée d'objets sonores palpables, clairement localisables dans l'espace (figure 6). L'utilisation d'un haut-parleur ultra-directif mobile et fixé au plafond de la passerelle du Musée d'Art des Grisons à Coire (Suisse) permet de projeter à même le sol de la passerelle le son de pas enregistrés préalablement. Telle une poursuite lumineuse dans un théâtre, une source sonore fantôme se promène sur le sol, comme si une personne invisible montait et descendait continûment cet espace qui se transforme en "salle des pas perdus". Les pas diffusés ayant été enregistrés tantôt sur du gravier ou de l'herbe, tantôt en extérieur avec des chaussures à talons ou en intérieur avec des chaussons, font référence à des représentations en mémoire partagées par la grande majorité des auditeurs. En traversant la passerelle, leur mémoire est réactivée et ils peuvent se surprendre à chercher une personne connue qui déambule à côté d'eux ou à se 


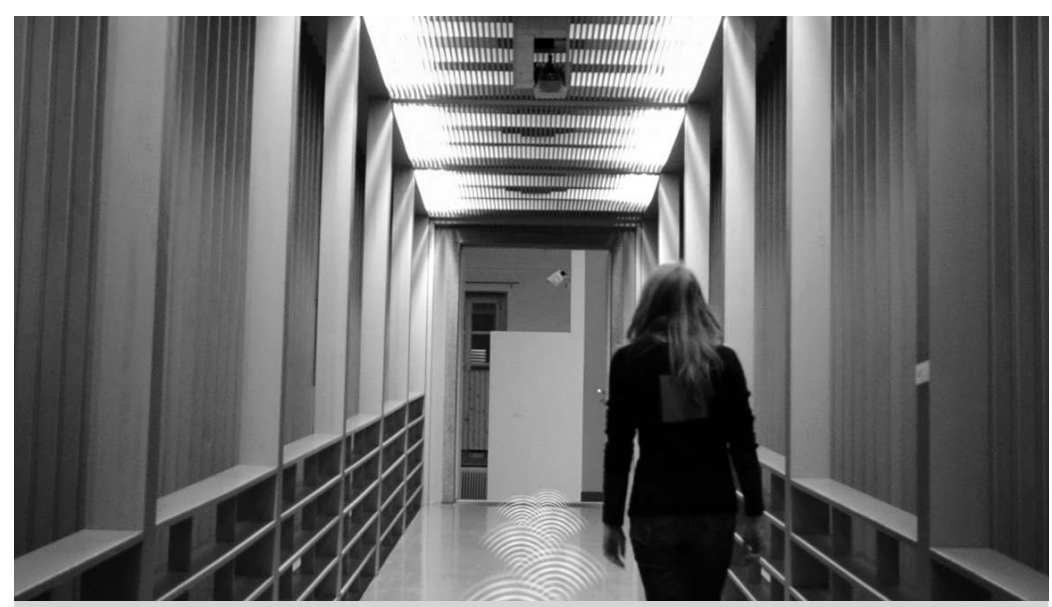

Figure 6 - "Wandelgang". Musée d'Art des Grisons, 2011, Coire, Suisse. Photo: Corsin Vogel.

projeter dans un autre lieu, comme le jardin du musée et ses allées de gravillons que l'on aperçoit depuis la passerelle.

Enfin, l'aspect palpable du son se découvre parfois de manière parfaitement inattendue, comme une révélation auditive. J'en ai fait l'expérience lors de mesures de bruit du Beluga, l'avion-cargo A300-600ST d'Airbus. Lors d'un passage de l'avion à basse altitude, moteurs coupés (le temps des mesures), on observait le stupéfiant passage silencieux de cet immense avion qui défie les lois de la pesanteur. Et tout à coup, quelques secondes après le survol de l'avion, des "spirales" sonores se matérialisèrent dans l'espace et se déplacèrent à toute allure tout autour de moi, tels de joyeux vifs et gros insectes. Si l'explication acoustique de vortex créés en bouts d'ailes est très plausible, la révélation auditive, elle, n'en a pas été moins inouie et poétique. C'est le genre d'expérience perceptive que je souhaite à chacune et à chacun, dans le silence d'un espace presque vide ou dans le brouhaha incessant d'une ville turbulente. Il suffit alors d'écouter.

\section{CONCLUSION}

Ces quelques expériences personnelles sur la sémantique des sons, leur spatialité et leur plastique, sans oublier leurs évolutions et évanescence temporelles, m’ont permis de suggérer au public, par l'intermédiaire d'installations sonores in situ, des écoutes très diversifiées. Il s'agit avant tout de donner à écouter autrement un quotidien où les sons, même les plus forts, sont souvent relégués sous une épaisse couche d'indifférence, quand ils ne sont pas rejetés et stigmatisés par les extrémistes du silence. Proposer de nouvelles formes d'écoute, 
réactiver des imaginaires propres à chacune et à chacun, laisser les auditeurs s'approprier les sons diffusés, laisser les écouteurs faire les tableaux, ${ }^{3}$ tels sont les objectifs de cette démarche artistique. Les projets ne manquent pas pour développer ces aspects passionnants de l'objet sonore, objet à la fois sémantique, temporel et spatial. Jouer avec ces trois caractéristiques offre une liberté immense pour sculpter les sons et suggérer du sens afin d'accéder auprès des auditeurs à une multitude de représentations en mémoire.

\section{BIBLIOGRAPHIE}

BAILBLE, Claude, 1998, "L'image frontale, le son spatial”, in Frank Beau, Philippe Dubois et Gérard Leblanc (dirs.), Cinéma et dernières technologies, Paris et Bruxelles, INA/De Boeck Université, 225-249.

CAUX, Daniel, 2009, "Luc Ferrari”, in Daniel Caux, Philippe Gras et Christian Rose, Le silence, les couleurs du prisme et la mécanique du temps qui passe. Paris, Editions de l'Eclat, 333-337.

DESHAYS, Daniel, 2004, "L'image sonore", conférence "Université de tous les savoirs", Université Paris Descartes, le 18 juillet 2004, en ligne <http://www.canal-u.tv/producteurs/universite_de_tous_les_savoirs/dossier_programmes/les_conferences_de_1_ annee_2004/image_fixe_image_mouvante/__image_sonore> (consulté le 02/10/2013).

DUBOIS, Danièle, 1993, Sémantique et cognition: Catégories, prototypes, typicalité. Sciences du langage, Paris, CNRS Editions.

DUBOIS, Danièle, 1998 [1993], "Comment l'homme communique-t-il?", in Annick WeilBarais (dir.), L'homme cognitif. Paris, Presses Universitaires de France, 199-301.

GIBSON, James J., 1986 [1979], The Ecological Approach to Visual Perception. Hillsdale, NJ, Lawrence Erlbaum Associates.

JOYCE, James, 1939, Finnegans Wake. Londres, Faber \& Faber.

MOORE, Anthony, 2010, "Transplacement", entretien avec Bill Fontana, in Peter Kiefer (dir.), Klangräume der Kunst. Heidelberg, Kehrer Verlag, 259-280.

ROSCH, Eleanor, 1978, "Principles of categorization", in Eleanor Rosch et Barbara L. Lloyd (dirs.), Cognition and Categorization. Hillsdale, NJ, Lawrence Erlbaum Associates, 27-48.

SCHAEFFER, Pierre, 1966, Traité des objets musicaux. Paris, Editions du Seuil.

THEILE, Günther, 1980, Untersuchung zur Wahrnehmung der Richtung und Entfernung von Phantomschallquellen bei 2-Kanal-Stereofonie. Munich, Institut für Rundfunktechnik.

VOGEL, Corsin, 1999, Etude sémiotique et acoustique de l'identification des signaux sonores d'avertissement en contexte urbain. Paris, Université Pierre et Marie Curie, Paris 6, thèse de doctorat, en ligne <http://www.lam.jussieu.fr/Publications/Theses/these-corsin-vogel.pdf $>$ (consulté le 02/10/2013).

3 En référence à Marcel Duchamp: "Ce sont les regardeurs qui font les tableaux". 